\title{
INDICADORES CIENTOMÉTRICOS DE PRODUÇÃO E COLABORAÇÃO CIENTÍFICA NA TEMÁTICA AGRADECIMENTOS (1980-2019).
}

\author{
SCIENTOMETRIC INDICATORS OF SCIENTIFIC \\ OUTPUT AND COLLABORATION ON THE \\ ACKNOWLEDGMENTS THEMATIC (1980-2019)
}

Gonzalo Rubén Alvareza

\begin{abstract}
RESUMO
Objetivo: Investiga-se as características da literatura mundial na temática dos agradecimentos do período de 1980-2019 indexada na base de dados Web of Science (WoS) através de indicadores cientométricos de produção e colaboração científica. Metodologia: Caracteriza-se como um estudo exploratório e descritivo que utilizou técnicas quantitativas (cientometria) para analisar todos os artigos abrangidos na categoria de assunto Information Science \& Library Science, com auxílio dos softwares Bibexcel e Pajek. Resultados: A distribuição dos 105 artigos sobre agradecimentos revela uma tendência de crescimento estável ao longo do período. O incremento significativo a partir de 2009 pode ter sido consequência do trabalho de captura e processamento automático de dados sobre agradecimentos iniciado pela WoS em 2008. Periódicos estrangeiros de língua inglesa lideram o ranking de produtividade e impacto, evidenciando-se a internacionalização e qualidade da produção científica. Os indicadores apontam duas gerações de pesquisadores na temática dos agradecimentos, o primeiro destacando-se em análises de uso, frequência e prevalência do tipo de apoio agradecido e o segundo em análises de informações sobre financiamento. Embora as coautorias identificadas nos artigos possam não representar um alto grau de conexão em rede, elas retratam vínculos colaborativos factuais de natureza intra-institucional. Conclusões: Conclui-se que as descobertas alcançadas até o momento simbolizam o esforço desses pesquisadores em um campo temático que não é novo, mas que está em transformação a partir do surgimento de novas oportunidades para obtenção de dados sobre agradecimentos.
\end{abstract}

" Artigo derivado da tese de doutorado "Práticas de agradecimento nos artigos científicos brasileiros indexados na Web of Science (2009-2016)" apresentada ao Programa de PósGraduação em Comunicação e Informação da Universidade Federal do Rio Grande do Sul (UFRGS).

a Doutor em Comunicação e Informação pela Universidade Federal do Rio Grande do Sul (UFRGS). Docente do Departamento de Ciência da Informação do Instituto de Arte e Comunicação Social da Universidade Federal Fluminense (UFF). E-mail: gonzalorubenalvarez@gmail.com. 
Descritores: Agradecimentos. Produção científica. Colaboração científica. Indicadores cientométricos. Cientometria.

\section{INTRODUÇÃO}

Os agradecimentos presentes em publicações representam atos de cortesia acadêmica (CRONIN; OVERFELT, 1994) e são decorrentes de interações e colaborações informais (e formais) entre atores sociais (indivíduos e instituições), tornando-se uma prática comum no processo de comunicação científica. O uso, frequência e tipo de agradecimento utilizado para recompensar formalmente os colaboradores variam conforme a disciplina e área, dependendo das necessidades, comportamento e estrutura de cada comunidade científica. Agradecimentos oferecem condições favoráveis para a geração de indicadores de colaboração de subautoria (HEFFNER, 1981) e representam influências intelectuais e materiais (financeiras e outras) daqueles que não adquiriram status de autor nos artigos.

Estudos sobre agradecimentos na ciência têm sido desenvolvidos sob a perspectiva da Sociologia da Ciência em nível de doutorado desde a década de 1970 (MACKINTOSH, $1972^{1}$ apud CRONIN; WEAVER, 1995). Devido à sua maior presença em artigos de periódicos, tal como observada em vários estudos, textos de agradecimentos se tornaram nas últimas décadas informações relevantes para explorar paralelamente padrões de colaboração e financiamento na ciência.

$\mathrm{Na}$ atualidade, o crescimento da ciência é fortemente vinculado a uma estrutura e organização social (comunidades científicas) que demanda não apenas colaboração, interação e relacionamento entre parceiros na busca de um mesmo objetivo científico, mas também recursos, investimentos e políticas de desenvolvimento institucional. Estudos sociológicos da ciência tornam-se fundamentais para interpretar a atividade científica, que há tempo deixou de ser realizada isoladamente, por um único cientista.

Na teoria de Merton (1988), os textos de agradecimentos, assim como as

1 MACKINTOSH, Kenneth Henry. Acknowledgement patterns in sociology. 1972. Tese (Doutorado) - University of Oregon, Oregon, 1972. 
citações, podem ser percebidos como resultado de relações sociais (formas de reconhecimento público, por pares qualificados, das contribuições científicas individuais) e que revelam aspectos simbólicos do sistema desigual de recompensa na ciência (Efeito Mateus), influenciado pela maior reputação, prestígio, autoridade e renome de cada cientista. O reconhecimento por pares qualificados é a forma básica de reconhecimento extrínseco na ciência, sendo que dele derivam todos os outros tipos de reconhecimentos, tais como o lucro econômico advindo de atividades científicas, o avanço na hierarquia acadêmica e o acesso ampliado ao capital científico humano e material (MERTON, 1988). O reconhecimento convencionado ou normativo é concedido não apenas quando um artigo é publicado (HAYASHI, 2020), mas também quando seu autor é citado ou agradecido formalmente por outra publicação.

Na doutrina de Kuhn (1997), o surgimento de revistas especializadas, a fundação de sociedades científicas, a constituição de currículos de cursos universitários, a publicação de livros, a referência a trabalhos anteriores (citações) e a inclusão de textos de agradecimento em artigos científicos podem ser consideradas formas de socialização e comunicação entre membros de uma disciplina que dividem as mesmas crenças, valores, técnicas e métodos, ou seja, que compartilham o mesmo paradigma. Na corrente sociológica de Ben-David (1975), o estudo dos agradecimentos como indicadores de colaboração se enquadraria no contexto das redes de comunicação científica e do reconhecimento dos diferentes tipos de contribuições dos pares durante 0 andamento das investigações no interior de comunidades (sistemas de interação fechados) com normas, sanções, lealdades e padrões de conduta próprios.

$\mathrm{Na}$ perspectiva de Knorr-Cetina (1982), textos de agradecimento a organismos de fomento à pesquisa, equipes técnicas e diretores podem ser considerados vestígios de interações e atividades que transcendem o laboratório - as arenas transepistêmicas ou campos transcientíficos, apresentando relações de cooperação e de dependência mútua em termos de recursos e suporte. $\mathrm{Na}$ teoria de Knorr-Cetina (1982), as relações entre cientistas e não cientistas expressas nos textos de agradecimento implicam escolhas e decisões técnicas, em que métodos a serem utilizados nas pesquisas são negociados com agências 
financiadoras e fornecedores de produtos (HOCHMAN, 1994).

$\mathrm{Na}$ visão de Latour e Woolgar (1997), os textos de agradecimento podem simbolizar práticas de colaboração e interação entre cientistas e/ou entre cientistas e órgãos de financiamento no espaço do laboratório, por exemplo, baseadas em relações sociais de caráter mercadológico e estratégico e de competição. O pensamento de Latour e Woolgar (1997) é contrário à teoria de Merton (1968), dado que este último considerava a comunidade científica como uma instituição social democrática, solidária, desinteressada e cooperativa.

Na perspectiva de Bourdieu (2004), textos de agradecimentos em artigos podem representar atos de reconhecimento (capital científico) que consistem no crédito e no valor atribuído por um ou mais cientistas concorrentes, sejam de colégios invisíveis ou não, no campo científico. Esses sinais de reconhecimento alimentam o capital científico (prestígio pessoal), podendo também indicar relações de colaboração e de estima mútua entre os pesquisadores envolvidos na investigação.

Em resumo, a presença de textos de agradecimentos em publicações pode revelar formas de socialização e comunicação baseadas em diferentes maneiras de organização e interação dos cientistas. Conforme as teorias discutidas, essas formas podem ser baseadas em normas e valores da comunidade (MERTON, 1968; BEN-DAVID, 1975; KUHN, 1997), em interesses e objetivos individuais (KNORR-CETINA, 1982; LATOUR; WOOLGAR, 1997; BOURDIEU, 2004).

No âmbito da Sociologia da Ciência e da Ciência da Informação, classificações para agradecimentos foram desenvolvidas por diversos autores. Inicialmente, dois grandes tipos de apoio de natureza não financeira foram individualizados por Patel (1973), os quais foram denominados por Heffner (1981) como technical aid e theoretical aid. O apoio técnico ou technical aid está relacionado com a coleta e processamento de dados, com o acesso e utilização de instalações e equipamentos de pesquisa e com a realização de análises estatísticas. $O$ apoio teórico ou theoretical aid está vinculado com a leitura, com a edição e com os comentários ao manuscrito original, resultante da pesquisa, antes da sua publicação. 
Cronin (1991), ao examinar a função social e o significado cognitivo dos agradecimentos presentes nos artigos publicados no Journal of the American Society for Information Science (JASIS) no período de 1970-1990, propôs uma tipologia mais detalhada da apresentada por Heffner (1981), que incluía seis categorias de agradecimento: technical, paymaster, dogsbody, moral support, prime mover, trusted assessor. De acordo com o Cronin (1991), a categoria technical indica o assessoramento sobre técnicas estatísticas, programas de computadores, processos experimentais e elaboração de instrumentos metodológicos; paymaster engloba o apoio financeiro, incluindo o recebimento de subsídios, incentivos e bolsas de estudo por parte do autor ou autores; dogsbody envolve o apoio administrativo, a orientação editorial e a assistência com a coleta de dados para análise; moral support abrange o apoio institucional, o acesso e a utilização de instalações (equipamentos, áreas de estudo, biblioteca), o apoio familiar, o acesso a informações relacionadas com pesquisas científicas; prime mover inclui o auxílio daqueles indivíduos que estimularam o desenvolvimento da pesquisa e a publicação dos resultados científicos; por último, a categoria trusted assessor contempla a ajuda daqueles colegas de trabalho que tiveram influência no artigo através das suas ideias, insights, feedback ou análise crítica.

McCain (1991) ao analisar os padrões de agradecimento identificados em pesquisas genéticas publicadas em 1988 no periódico Genetics desenvolveu um esquema de classificação com cinco categorias de agradecimentos: technical assistance (apoio vinculado a análises e procedimentos técnicos específicos envolvendo dados de pesquisa); manuscript preparation (assistência pósanalítica relacionada com a digitação do manuscrito, editoração de ilustrações, tabelas etc.); access to research-related information (suporte institucional associado com o acesso a materiais experimentais, tecnologia, instalações, protocolos não publicados etc.); access to unpublished results/data (ajuda relacionada com o acesso a dados não publicados); peer interactive communication (apoio intelectual e/ou conceitual dos pares durante a elaboração do manuscrito).

Uma versão modificada da tipologia desenvolvida por Cronin (1991) foi 
elaborada posteriormente por Cronin, McKenzie e Rubio (1993), a qual incluía as seguintes categorias de agradecimentos: technical support; financial support; clerical support (apoio relacionado com a digitação de dados, com a preparação editorial do manuscrito etc.); access (suporte institucional relativo ao acesso a instalações, dados não publicados, amostras, documentos, materiais etc.); moral support; peer interactive communication (PIC).

Em estudos mais recentes sobre a evolução e a importância funcional e simbólica dos agradecimentos presentes em dois periódicos das áreas de Ciências Sociais e Humanidades (CRONIN; SHAW; LA BARRE, 2003) e em um da área de Química (CRONIN; SHAW; LA BARRE, 2004), os tipos de reconhecimento para cada artigo analisado foram classificados da seguinte forma: instrumental/technical (suporte vinculado ao acesso de ferramentas, tecnologias, instalações e recursos de infraestrutura, fornecendo conhecimentos técnicos, tais como coleta de dados, desenho experimental ou análises estatísticas); financial (apoio financeiro interno e externo); editorial (assistência sobre preparação e apresentação de manuscritos, auxílio com pesquisas bibliográficas etc.); moral (apoio familiar, de amigos etc.); conceptual (orientação intelectual, insight crítico, influência ideológica de indivíduos que serviram de fonte de inspiração); unknown (apoio agradecido de natureza indefinida).

A categoria conceptual, similar às tipologias prime mover e trusted assessor propostas por Cronin (1991), foi inicialmente descrita por McCain (1991) como peer interactive communication (PIC), podendo ser considerada um tipo especial de agradecimento que envolve a contribuição intelectual e a influência de colegas do mesmo nível hierárquico. O caso da categoria PIC ou conceptual e do papel que desempenha é particularmente relevante, a tal ponto de que alguns especialistas consideram que o registro da influência recebida dos pares ou dívida intelectual, através dos agradecimentos, é comparável com o registro da influência através de citações (CRONIN; MCKENZIE; STIFFLER, 1992; CRONIN; MCKENZIE; RUBIO, 1993; DÍAZ-FAES; BORDONS, 2017).

Apesar das suas diferenças que, em parte, refletem diferenças na forma como o conhecimento é construído e utilizado de uma disciplina ou campo para outro (CRONIN; MCKENZIE; STIFFLER, 1992), as classificações para 
agradecimentos apresentadas no Quadro 1 têm muito em comum. É válido argumentar que estudos posteriores utilizaram outras categorias para classificar os agradecimentos (HAYASHI; BELLO, 2014; HAYASHI, 2018).

Quadro 1 - Esquemas de classificação para agradecimentos

\begin{tabular}{|c|c|c|c|c|}
\hline Heffner (1981) & McCain (1991) & Cronin (1991) & $\begin{array}{l}\text { Cronin, McKenzie } \\
\text { e Rubio (1993) }\end{array}$ & $\begin{array}{c}\text { Cronin, Shaw } \\
\text { e La Barre } \\
(2003,2004) \\
\end{array}$ \\
\hline \multirow[t]{4}{*}{ Technical aid } & $\begin{array}{l}\text { Technical } \\
\text { assistance }\end{array}$ & Technical & Technical support & $\begin{array}{l}\text { Instrumental/ } \\
\text { technical }\end{array}$ \\
\hline & & Paymaster & Financial support & Financial \\
\hline & $\begin{array}{l}\text { Manuscript } \\
\text { preparation }\end{array}$ & Dogsbody & Clerical support & Editorial \\
\hline & $\begin{array}{l}\text { Access to research- } \\
\text { related information } \\
\text { Access to } \\
\text { unpublished } \\
\text { results/data }\end{array}$ & Moral support & $\begin{array}{l}\text { Access } \\
\text { Moral support }\end{array}$ & Moral \\
\hline Theoretical aid & $\begin{array}{l}\text { Peer interactive } \\
\text { communication } \\
\text { (PIC) }\end{array}$ & $\begin{array}{l}\text { Prime mover } \\
\text { Trusted } \\
\text { assessor }\end{array}$ & $\begin{array}{l}\text { Peer interactive } \\
\text { communication } \\
\text { (PIC) }\end{array}$ & Conceptual \\
\hline & & & & Unknown \\
\hline
\end{tabular}

Fonte: Heffner (1981), McCain (1991), Cronin (1991), Cronin, McKenzie e Rubio (1993), Cronin, Shaw e La Barre $(2003,2004)$

Portanto, a pesquisa busca analisar as características da literatura mundial na temática dos agradecimentos do período de 1980-2019 indexada na base de dados Web of Science (WoS) através de indicadores cientométricos de produção e colaboração. Os indicadores a serem apresentados pretendem evidenciar a evolução temporal das publicações, a escolha dos periódicos utilizados para veiculação das descobertas científicas, a contribuição em nível de autores, instituições e países e a colaboração entre indivíduos. Os resultados decorrentes das análises podem propiciar um ambiente favorável para discutir políticas científicas que visem à ampliação das pesquisas sobre agradecimentos, principalmente no âmbito nacional. 


\section{PROCEDIMENTOS METODOLÓGICOS}

O corpus deste estudo cientométrico é constituído pelos artigos sobre agradecimentos do período de 1980-2019 indexados na base de dados multidisciplinar WoS. A busca e coleta dos registros bibliográficos foram realizadas em 25 de outubro de 2020, através da opção de pesquisa avançada, nos índices Science Citation Index Expanded (SCI-EXPANDED), Social Sciences Citation Index (SSCl), Arts \& Humanities Citation Index (A\&HCl) e Emerging Sources Citation Index (ESCl), incluindo todos os idiomas.

A escolha do artigo como unidade de análise justifica-se pelo fato de ser normalmente o tipo de documento que recebe maior atenção da comunidade científica, refletindo-se no número de citações que recebe em relação aos demais. A combinação de índices ampliou as possibilidades de localização de registros bibliográficos. A delimitação temporal ressalta a abrangência da pesquisa, analisando a evolução das publicações sobre agradecimentos em um período de 40 anos.

$\mathrm{Na}$ recuperação dos artigos, utilizou-se o rótulo de campo TS=Topic. Dessa maneira, foi possível pesquisar por termos/expressões no título, resumo, palavras-chave de autor e palavras-chave da base de dados (KeyWords Plus) das publicações. Utilizou-se o rótulo de campo WC=Web of Science Category para delimitar os artigos sobre agradecimentos em periódicos indexados pela WoS nas áreas da Biblioteconomia e Ciência da Informação. Com uso de operadores booleanos, a estratégia de busca foi definida da seguinte maneira: $\mathrm{TS}=\left(\right.$ acknowledg $\left.^{*}\right)$ AND WC $=($ Information Science \& Library Science $)$.

Posteriormente, os dados foram importados em um único arquivo de texto (.txt), removendo os registros duplicados com auxílio do software Bibexcel. Os nomes dos autores, instituições e países foram manualmente padronizados para geração das frequências. Os 105 registros bibliográficos identificados foram analisados a partir de indicadores cientométricos de produção (ano de publicação, idioma, periódico, produtividade de autores, instituições e países) e de colaboração (coautoria entre autores). A relação entre produtividade e impacto através dos artigos mais citados por periódico e autor foi estimada com 
auxílio do $h$-index (HIRSCH, 2005).

O Bibexcel é um software livre e gratuito, de uso acadêmico, e foi utilizado na organização dos dados bibliográficos e geração das frequências por cada variável analisada. O Microsoft Excel possibilitou a elaboração de tabelas e figuras. O software Pajek foi utilizado para representar a rede de colaboração entre os autores mais produtivos. Esta pesquisa utilizou a contagem total, isto é, um artigo ou citação para cada autor, instituição ou país que assinou a publicação

\section{RESULTADOS E DISCUSSÃO}

A Figura 1 apresenta a distribuição das 105 publicações sobre a temática "agradecimentos" de 1980-2019 indexadas pela WoS. De modo geral, observase uma tendência de crescimento estável ao longo do período analisado $\left(R^{2}=0,7536\right)$.

O baixo número de trabalhos publicados nas décadas iniciais pode ser um reflexo das limitações metodológicas, que envolviam, naquela época, a identificação e coleta de dados sobre agradecimentos para análise. Portanto, algumas das primeiras investigações no assunto compreenderam corpus de dados e/ou intervalos de tempo relativamente pequenos. No contexto da Sociologia da Ciência e da Ciência da Informação, no começo, os agradecimentos foram um assunto relativamente negligenciado em virtude da dificuldade para coletar manualmente informações dos artigos (CRONIN; SHAW; LA BARRE, 2003; HYLAND, 2003; GILES; COUNCILL, 2004).

Por meio de dados sobre agradecimentos, o primeiro artigo publicado no período de 1980-2019 demonstrou que, em disciplinas como Biologia, Ciência Política, Psicologia e Química, o apoio financeiro está associado ao aumento no número total de colaboradores envolvidos na produção científica, compreendendo coautores e subautores (indivíduos agradecidos por apoio técnico e/ou intelectual/conceitual) (HEFFNER, 1981).

Nas décadas de 1990 e 2000 (até 2006), pesquisadores internacionais exploraram a função e o significado social e cognitivo das práticas de agradecimentos na ciência por disciplina e área através do mapeamento da 
frequência de uso e prevalência dos tipos de apoio agradecidos em publicações, destacando-se os trabalhos de Blaise Cronin e colaboradores. Por exemplo, observou-se que quase a metade dos artigos publicados em JASIS contém agradecimentos do tipo trusted assessor (CRONIN, 1991), ressaltando a importância dessa categoria para identificar e mapear redes de influência conceitual e/ou intelectual na ciência. Naquela época, Cronin (1991) já sugeria que, devido à sua potencialidade, os agradecimentos fossem utilizados conjuntamente com as citações como um indicador de qualidade/impacto na avaliação do desempenho da atividade científica.

A posteriori, em análise dos agradecimentos do tipo PIC, evidenciou-se que as práticas variam entre periódicos de uma mesma área, sendo o caso da Biblioteconomia \& Ciência da Informação (CRONIN; MCKENZIE; STIFFLER, 1992). Os autores citados constataram uma correlação positiva entre a frequência de agradecimentos e citações para indivíduos altamente agradecidos pela contribuição intelectual durante 0 andamento da pesquisa. O predomínio de agradecimentos do tipo PIC e a importância do feedback e da análise crítica dos pares durante o desenvolvimento do manuscrito também foi constatado em publicações da Psicologia, Sociologia, História e Filosofia, apontando comportamentos similares entre áreas (CRONIN; MCKENZIE; RUBIO, 1993).

Em análise dos agradecimentos na literatura da Ciência da Informação, anos mais tarde, concebeu-se a ideia de que a intensificação do uso dos agradecimentos nos artigos da área seja, em certa medida, um sinal de amadurecimento da comunidade científica a respeito do significado instrumental e normativo dessa prática (CRONIN, 2001). Ao examinar a frequência de uso e a prevalência do tipo de apoio agradecido na literatura de diferentes disciplinas, evidenciou-se que a supremacia de textos de agradecimentos do tipo financial em Psicologia e PIC em Filosofia refletem diferenças interdisciplinares em termos de estrutura sociocognitiva e método de trabalho (CRONIN; SHAW; LA BARRE, 2003). O predomínio de artigos com manifestações de gratidão na categoria financial da Química pode representar a dependência do apoio financeiro externo em campos experimentais (CRONIN; SHAW; LA BARRE, 2004). 
A partir de 2009 e decorrer da década de 2010, observa-se um importante crescimento do número de publicações, provavelmente, como consequência do trabalho de identificação, captura e processamento automático de dados sobre agradecimentos por apoio financeiro realizado pela WoS (e outras bases) em registros bibliográficos indexados em SCIE desde 2008 e SSCI desde 2015 (PAUL-HUS et al., 2015; PAUL-HUS; DESROCHERS; COSTAS, 2016). Desde então, a disponibilização de dados sobre agradecimentos identificados em artigos de revistas científicas por parte de bases de dados bibliográficas abriu novas possibilidades para explorar não apenas aspectos inerentes à colaboração científica de subautoria, mas também ao financiamento na pesquisa desde uma perspectiva mais ampla. Estudos sobre financiamento na atividade científica via agradecimentos são desenvolvidos com maior intensidade e complexidade desde a década de 1990, destacando-se os trabalhos de Grant Lewison e colaboradores. $O$ fato é que as publicações sobre agradecimentos do período de $2009-2019$ representam quase $70,0 \%$ do total de artigos analisados.

Figura 1 - Evolução da produção científica sobre agradecimentos na WoS (19802019)

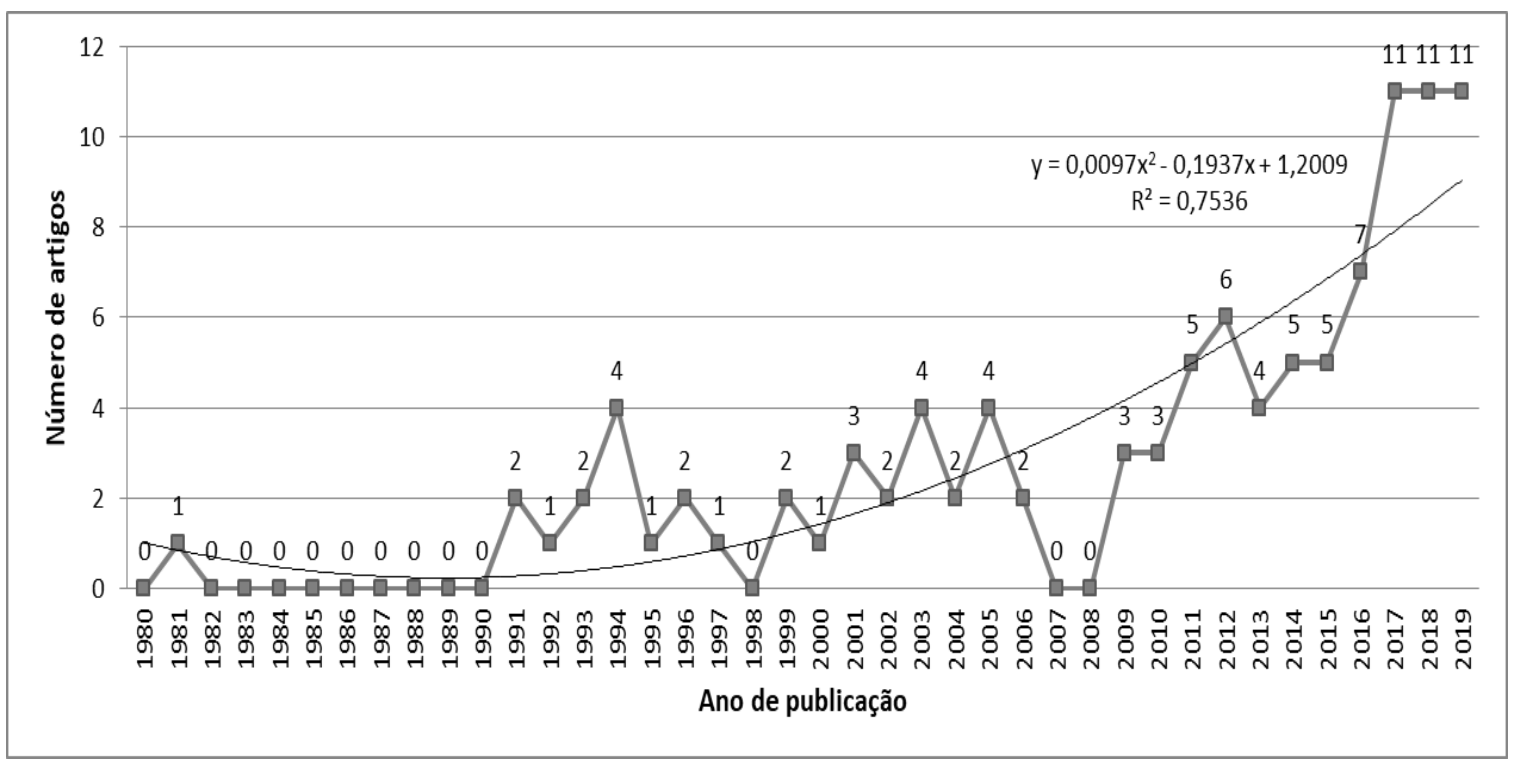

Fonte: Web of Science (2020)

A distribuição dos 105 artigos sobre agradecimentos por idioma revelou que $97,0 \%$ das publicações foram escritas em inglês, ou seja, no idioma oficial da ciência. Esse resultado deve ser analisado com cautela, uma vez que a WoS 
prioriza a indexação de revistas na língua inglesa, visando atingir, dessa maneira, uma grande audiência para discussão de resultados de pesquisas. Tal política de indexação de periódicos na WoS pode causar, consequentemente, uma baixa cobertura, no âmbito internacional, de revistas nacionais que publicam em línguas diferentes ao inglês. O fato é que, a ausência de idiomas diferentes ao inglês nas publicações pode sinalizar uma carência de estudos sobre a temática dos agradecimentos, principalmente, nos países da América Latina. Embora presentes na literatura internacional em inglês, estudos sobre agradecimentos no processo de comunicação científica não encontraram ainda um espaço consolidado na agenda de pesquisa de países como o Brasil (HAYASHI; BELLO, 2014). Sendo assim, torna-se necessário a inclusão dos agradecimentos como temática de pesquisa no âmbito nacional, visando ampliar a compreensão do significado da colaboração de subautoria e o impacto do financiamento na ciência regional.

A distribuição dos 105 artigos sobre agradecimentos por periódico mostra uma baixa dispersão da produção indexada na WoS, uma vez que foram utilizados apenas 30 veículos de comunicação para divulgar as descobertas científicas. $O$ desenvolvimento progressivo das pesquisas observado na Figura 1 através do número de artigos publicados anualmente pode indicar um aumento do interesse na temática no decorrer do tempo, sendo que periódicos editados nos Estados Unidos e Inglaterra foram responsáveis pela publicação de 60 artigos $(57,1 \%)$, ou seja, mais da metade do total de publicações sobre agradecimentos. A supremacia de agradecimentos em revistas inglesas e americanas sugere que essa prática não é tão comum fora do contexto científico anglo-americano, estando longe de ser universalmente aceita, apesar de ter sido observado na atualidade um incremento em revistas não anglófonas (SALAGERMEYER; ARIZA; BERBESÍ, 2009; SALAGER-MEYER et al., 2011). As constatações dos autores supracitados referentes à frequência de uso dos agradecimentos evidenciam a interdependência acadêmica e o comportamento colaborativo em rede da ciência anglo-americana, podendo justificar a preferência dos pesquisadores para publicarem seus artigos em revistas inglesas e americanas. 
Torna-se evidente a internacionalização da produção científica sobre agradecimentos, mensurada pela publicação de artigos em periódicos estrangeiros e pelo uso do idioma inglês, já que foi identificado apenas um artigo publicado em periódico editado na América Latina. Em termos de volume de publicações, o protagonismo dos principais periódicos científicos nas áreas de Biblioteconomia e Ciência da Informação, tais como Scientometrics, Journal of the Association for Information Science and Technology (JASIST), Journal of Documentation e Research Evaluation, pode ser claramente percebido na Tabela 1. Os quatro periódicos apresentam alto fator de impacto $(F I)$ e concentram quase $60,0 \%$ do total da literatura sobre agradecimentos publicada no período de 1980-2019. A relação entre produtividade e impacto das publicações por periódico foi estimada com auxílio do $h$-index. Os indicadores mostram que os veículos com maior número de artigos publicados receberam maior número de citações, indicando a visibilidade e qualidade das pesquisas divulgadas nesses canais de comunicação. De modo geral, percebe-se uma concentração das publicações sobre agradecimentos no âmbito dos estudos métricos da informação, utilizando métodos quantitativos para analisar características da colaboração e da pesquisa financiada na ciência.

Tabela 1 - Periódicos da produção científica sobre agradecimentos na WoS (1980-2019)

\begin{tabular}{|c|c|c|c|c|c|c|}
\hline \multirow[b]{2}{*}{ Periódico } & \multirow[b]{2}{*}{ País } & \multicolumn{2}{|c|}{ Produção } & \multicolumn{3}{|c|}{ Impacto } \\
\hline & & Artigos & $\%$ & h-index & $\begin{array}{l}\text { Citações } \\
\text { (h-core) }\end{array}$ & $\begin{array}{c}\text { Total } \\
\text { citações }\end{array}$ \\
\hline Scientometrics & Holanda & 30 & 28,6 & 12 & 447 & 563 \\
\hline $\begin{array}{l}\text { Journal of the Association for } \\
\text { Information Science and } \\
\text { Technology }\end{array}$ & EUA & 16 & 15,2 & 14 & 801 & 821 \\
\hline Journal of Documentation & Inglaterra & 8 & 7,6 & 7 & 208 & 210 \\
\hline Research Evaluation & Inglaterra & 7 & 6,7 & 6 & 318 & 318 \\
\hline $\begin{array}{l}\text { Aslib Journal of Information } \\
\text { Management }\end{array}$ & Inglaterra & 5 & 4,8 & 4 & 23 & 25 \\
\hline Journal of Scholarly Publishing & Canadá & 4 & 3,8 & 2 & 18 & 18 \\
\hline Journal of Information Science & Inglaterra & 3 & 2,9 & 3 & 117 & 117 \\
\hline $\begin{array}{l}\text { Library \& Information Science } \\
\text { Research }\end{array}$ & EUA & 3 & 2,9 & 3 & 24 & 24 \\
\hline Learned Publishing & Inglaterra & 2 & 1,9 & 2 & 10 & 10 \\
\hline $\begin{array}{l}\text { Journal of the Canadian Health } \\
\text { Libraries Association }\end{array}$ & Canadá & 2 & 1,9 & 2 & 9 & 9 \\
\hline
\end{tabular}




\begin{tabular}{lllllll}
\hline $\begin{array}{l}\text { Journal of Academic } \\
\text { Librarianship }\end{array}$ & EUA & 2 & 1,9 & 2 & 15 & 15 \\
\hline Publications & Suíça & 2 & 1,9 & 1 & 6 & 7 \\
\hline $\begin{array}{l}\text { Journal of Scientometric } \\
\text { Research }\end{array}$ & Índia & 2 & 1,9 & 1 & 9 & 10 \\
\hline $\begin{array}{l}\text { Information Research - An } \\
\text { International Electronic Journal }\end{array}$ & Inglaterra & 2 & 1,9 & 1 & 5 & 5 \\
\hline $\begin{array}{l}\text { Collnet Journal of } \\
\text { Scientometrics and Information } \\
\text { Management }\end{array}$ & Inglaterra & 2 & 1,9 & 0 & 0 & 0 \\
\hline
\end{tabular}

Fonte: Web of Science (2020)

A produção científica sobre agradecimentos, constituída por 105 artigos, de 1980-2019 e indexada na WoS envolve a contribuição de 153 autores de diversas nacionalidades. Cada publicação tem em média 1,5 autores. A autoria única é predominante e representa $37,1 \%$ do total de publicações. A maior parte das produções concentra poucos autores por artigo, o que é normal em disciplinas de áreas como, Ciências Sociais e Humanidades, cujos níveis de coautoria costumam ser baixos. $O$ fato é que artigos assinados por até 3 autores representam $88,1 \%$. Diferentes motivações pessoais e profissionais parecem ter um papel importante na formação de redes de colaboração, podendo estar relacionadas com a natureza da pesquisa (teórica ou experimental) praticada em cada área e com a obtenção de financiamento.

Blaise Cronin filiado à Indiana University apresenta o $h$-index mais alto (Tabela 2), evidenciando a excelência e o impacto da produção científica sobre agradecimentos desse pesquisador no âmbito da Ciência da Informação, Bibliometria e Cientometria. Pioneiro no assunto, Cronin tem desenvolvido estudos quali-quantitativos desde início da década de 1990, explorando características textuais e funcionais dos agradecimentos inseridos no processo tradicional de comunicação e produção científica. Em análise da produção científica sobre agradecimentos, comprovou-se que a distribuição do número de pesquisadores pelo número de artigos segue uma linha de tendência de potência ( $\left.R^{2}=0,9044\right)$, em concordância com o modelo proposto pela Lei de Lotka ou lei de produtividade de autores. Na maioria das áreas, onde o número de coautores nas publicações é sempre limitado, o modelo do quadrado inverso continua vigente. No entanto, em áreas altamente colaborativas como, por exemplo, a Física de Altas Energias (FAE), foi comprovada a inaplicabilidade da lei 
(KRETSCHMER; ROUSSEAU, 2001).

Tabela 2 - Pesquisadores mais produtivos nas publicações sobre agradecimentos na WoS (1980-2019)

\begin{tabular}{|c|c|c|c|c|c|}
\hline \multirow[b]{2}{*}{ Pesquisador } & \multirow[b]{2}{*}{ Instituição } & \multicolumn{2}{|l|}{ Produção } & \multicolumn{2}{|l|}{ Impacto } \\
\hline & & Artigos & h-index & $\begin{array}{c}\text { Citações } \\
\text { (h-core) }\end{array}$ & $\begin{array}{c}\text { Total } \\
\text { citações }\end{array}$ \\
\hline Cronin, B. & Indiana Univ. & 16 & 14 & 811 & 823 \\
\hline Lewison, G. & City, Univ. London & 9 & 7 & 117 & 121 \\
\hline Desrochers, N. & Univ. Montreal & 5 & 5 & 69 & 69 \\
\hline Morillo, F. & CSIC & 5 & 4 & 34 & 37 \\
\hline Bordons, M. & $\mathrm{CSIC}$ & 5 & 3 & 51 & 56 \\
\hline Paul-Hus, A. & Univ. Montreal & 4 & 4 & 77 & 77 \\
\hline Pecoskie, J. & Wayne State Univ. & 4 & 4 & 31 & 31 \\
\hline Rigby, J. & Univ. Manchester & 4 & 4 & 103 & 103 \\
\hline Costas, R. & Leiden Univ. & 4 & 3 & 108 & 111 \\
\hline Shaw, D. & Indiana Univ. & 3 & 3 & 269 & 269 \\
\hline McKenzie, G. & Indiana Univ. & 3 & 3 & 94 & 94 \\
\hline Díaz-Faes, A. A. & $\mathrm{CSIC}$ & 3 & 2 & 34 & 36 \\
\hline Sugimoto, C. R. & Indiana Univ. & 3 & 2 & 30 & 30 \\
\hline Hartley, J. & Univ. Keele & 3 & 2 & 19 & 19 \\
\hline Álvarez-Bornstein, B. & CSIC & 3 & 2 & 23 & 25 \\
\hline Van Leeuwen, T. N. & Leiden Univ. & 2 & 2 & 67 & 67 \\
\hline Tan, A. M. & Zhejiang Univ. & 2 & 2 & 16 & 16 \\
\hline Shapira, P. & Univ. Manchester & 2 & 2 & 89 & 89 \\
\hline Mongeon, $\mathrm{P}$. & Univ. Montreal & 2 & 2 & 33 & 33 \\
\hline Larivière, $\mathrm{V}$. & Univ. Montreal & 2 & 2 & 33 & 33 \\
\hline McCain, K. W. & Drexel Univ. & 2 & 2 & 20 & 20 \\
\hline La Barre, K. & Indiana Univ. & 2 & 2 & 232 & 232 \\
\hline Salager-Meyer, F. & Univ. Los Andes & 2 & 2 & 35 & 35 \\
\hline Rubio, L. & Indiana Univ. & 2 & 2 & 60 & 60 \\
\hline Zhao, S. X. & Univ. Alberta & 2 & 2 & 16 & 16 \\
\hline
\end{tabular}

Fonte: Web of Science (2020)

Os pesquisadores que publicaram na temática dos agradecimentos estão vinculados a 91 instituições. A Indiana University ocupa uma posição de privilégio, uma vez que detém 17 artigos, representando $16,2 \%$ da produção total. A hegemonia das instituições americanas e inglesas no ranking de produtividade não é novidade, levando em consideração a procedência dos autores pioneiros no assunto. Resulta interessante destacar a contribuição de instituições prestigiosas nos estudos métricos da informação como o Consejo 
Superior de Investigaciones Científicas (CSIC) (7,6\%), Université de Montréal $(5,7 \%)$ e Leiden University $(4,8 \%)$ na produção científica.

Os artigos sobre agradecimentos foram assinados por pesquisadores de diversas partes do mundo, no entanto, observa-se uma baixa dispersão da produção por país. Cientistas de apenas 27 países publicaram trabalhos sobre agradecimentos indexados pela WoS até 2019. É indiscutível o protagonismo dos Estados Unidos e Inglaterra, dado que juntos detêm quase $60,0 \%$ da produção internacional, confirmando a teoria de que os agradecimentos como objeto de estudo é e sempre foi um fenômeno social anglo-americano (SALAGER-MEYER et al., 2011). O bom posicionamento no ranking de produtividade da Espanha (12,4\%), Canadá (9,5\%) e Holanda (4,8\%) não é surpreendente, já que esses países estão bem representados no ranking institucional.

A rede de coautoria dos autores das publicações sobre agradecimentos indexadas pela WoS é apresentada na Figura 2. No grafo, os vértices representam a comunidade científica internacional e as arestas os artigos em colaboração. De modo a evitar a sobreposição, alguns vértices foram moderadamente reposicionados dentro do grafo principal. A estrutura do grafo reflete o "companheirismo" intelectual (KATZ; MARTIN, 1997) e as relações de parceria na ciência e está constituída por 128 autores, destacando-se o pesquisador Blaise Cronin da Indiana University (EUA) como o ator mais popular na rede de colaboração dos autores mais produtivos. 
Figura 2 - Rede de colaboração dos pesquisadores mais produtivos nos artigos sobre agradecimentos na WoS (1980-2019)

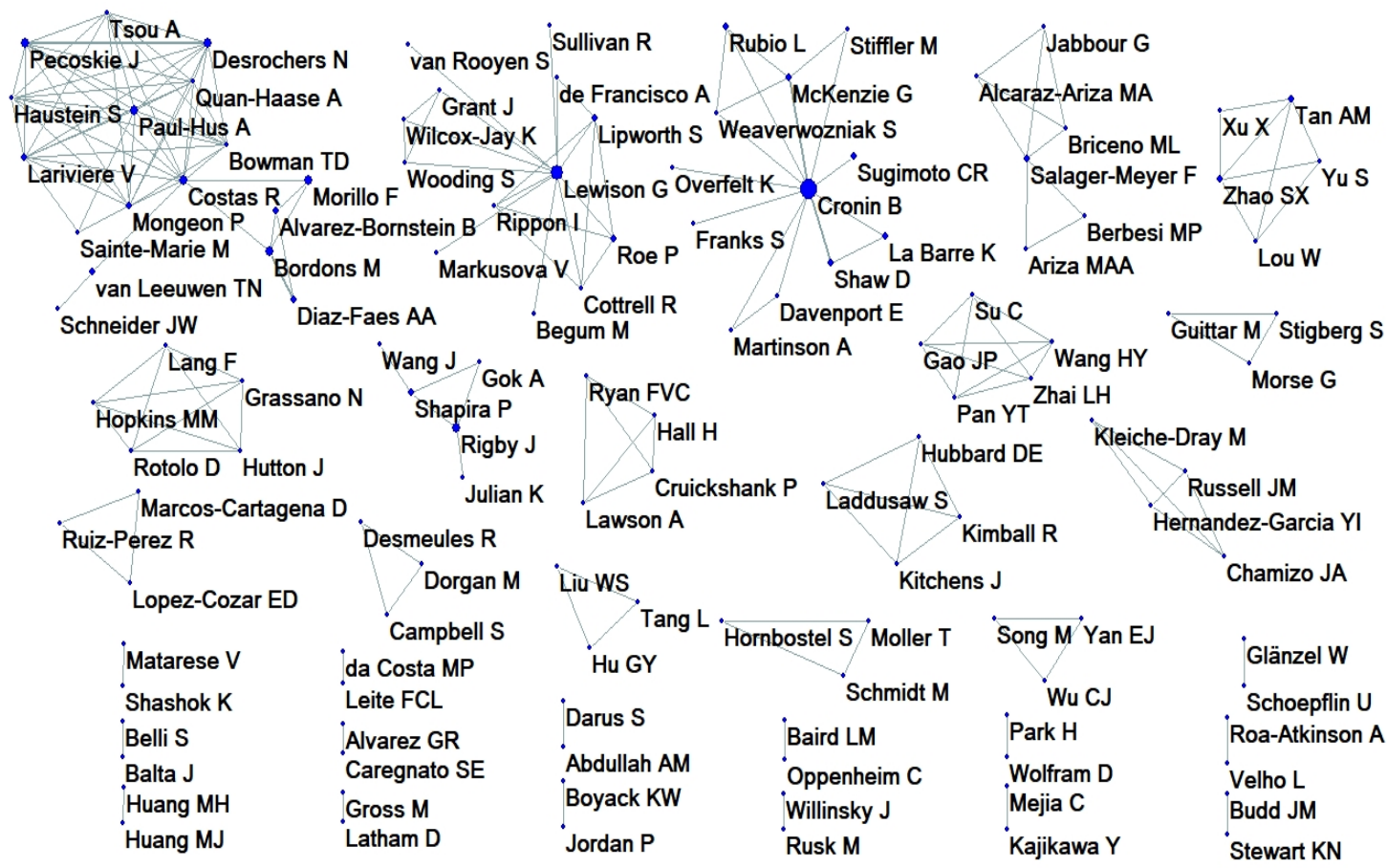

Fonte: Web of Science (2020)

A respeito da densidade da rede de coautoria em nível de indivíduos, observam-se alguns clusters na parte superior da estrutura do grafo, evidenciando vínculos colaborativos factuais entre pesquisadores filiados a uma mesma instituição. Um dos clusters é constituído por pesquisadores vinculados à Indiana University, incluindo o autor mais produtivo no período de 1980-2019 (Cronin B., Shaw D., La Barre K., Sugimoto C., Overfelt K., Rubio L., Stiffler M.). Tal indicador reforça a teoria de que a proximidade geográfica é um fator que estimula a colaboração entre dois ou mais parceiros e, consequentemente aumenta as chances de publicar trabalhos em coautoria (KATZ; MARTIN, 1997). $\mathrm{Na}$ atividade científica parece haver um consenso de que a maioria dos pesquisadores colabora mais com aqueles que fazem parte do seu ambiente cotidiano (BOZEMAN; LEE, 2003). As crenças pessoais, a cultura, a língua, os costumes e as questões políticas são também fatores influenciadores que afetam o nível de intensidade da colaboração (KATZ, 1994).

Outro dos clusters está constituído por pesquisadores filiados à Université de Montréal (Desrochers N., Paul-Hus A., Haustein S., Larivière P., Mongeon P.). 
O nível de agrupamento neste cluster é destacável, oferecendo condições favoráveis para entender como se constituem as comunidades a partir da troca de informações e da produção conjunta de conhecimento. O pesquisador Costas R. (Leiden University) ocupa uma posição de privilégio na rede, uma vez que aproxima os cientistas da Université de Montréal com os do CSIC (Bordons M., Morillo F., Álvarez-Bornstein B., Díaz-Faes A.), estimulando a formação de parcerias com colegas de diversas instituições interessados na temática dos agradecimentos. A colaboração não só afeta à produtividade e à visibilidade, mas também à obtenção de financiamento (BOZEMAN; LEE, 2003), necessário para o desenvolvimento das pesquisas.

Embora presentes na literatura internacional, os estudos sobre agradecimentos na América Latina são escassos e incipientes, merecendo uma maior atenção da região para explorar características da colaboração "oculta" (agradecimentos não financeiros) e da pesquisa financiada (agradecimentos financeiros). A predominância de autores estrangeiros na rede de coautoria traz à tona a baixa cobertura dos agradecimentos como campo temático de investigação em países como o Brasil. Levando em consideração as publicações indexadas na categoria de assunto Information Science \& Library Science da WoS, destaca-se a iniciativa dos pesquisadores brasileiros filiados à Universidade Federal do Rio Grande do Sul (UFRGS).

\section{CONSIDERAÇÕES FINAIS}

Através da análise dos 105 registros bibliográficos que constituíram o corpus desta pesquisa foi possível caracterizar a literatura sobre agradecimentos do período de 1980-2019 indexada na WoS, utilizando indicadores cientométricos de produção e colaboração científica. Apesar do desenvolvimento tardio e o baixo número de artigos na década inicial, de modo geral, observa-se uma evolução temporal das publicações mundiais. A realidade mostra que houve um incremento significativo das publicações a partir de 2009, logo depois de que bases de dados como a WoS começassem a coletar e processar sistematicamente informações sobre agradecimentos dos artigos. Certamente, a iniciativa da WoS pode ter incentivado diversos cientistas para ampliarem os 
estudos sobre agradecimentos como indicadores de subautoria e financiamento. O fato é que, ainda é necessário melhorar não apenas a compreensão dos agradecimentos como fenômeno social no âmbito acadêmico, mas também a qualidade dos dados de pesquisa utilizados nos estudos métricos da informação. Nesse sentido, autores, periódicos e bases de dados têm uma responsabilidade enorme, ao gerarem, registrarem e processarem os textos sobre agradecimentos decorrentes de múltiplos tipos de interações.

A prevalência do idioma inglês e dos periódicos estrangeiros nas publicações pode refletir a internacionalização da pesquisa sobre agradecimentos. Por outro lado, pode expor algumas limitações da WoS em termos de cobertura. De qualquer maneira, os baixos índices de literatura em outros idiomas podem simbolizar um desafio para as comunidades científicas cuja língua nativa não é o inglês no campo temático de investigação, sendo o caso dos países da América Latina. Os principais periódicos em Biblioteconomia e Ciência da Informação se destacam no ranking de produtividade e impacto (número de citações), denotando a qualidade da pesquisa sobre agradecimentos divulgada nesses veículos de comunicação.

Os indicadores de produção científica em nível de indivíduos apontam duas gerações de pesquisadores. Liderada por B. Cronin, a primeira se destacou pelas análises de uso, frequência e prevalência do tipo de apoio formalmente agradecido nos artigos, explorando padrões de subautoria por disciplina e área. Constituída a partir de 2009, a segunda geração é consequência das ações desenvolvidas pela WoS e inclui um número maior de pesquisadores, explorando, através dos agradecimentos por financiamento, diversos elementos da pesquisa financiada. Trabalhos recentes têm buscado compreender a completude e a precisão da WoS na extração e processamento de dados sobre financiamento via agradecimentos (ÁLVAREZ-BORNSTEIN; MORILLO; BORDONS, 2017).

Por meio dos indicadores cientométricos de produção em nível de instituições, os agradecimentos apresentam-se como um assunto de pesquisa interessante dentro de grupos de pesquisa institucionais reconhecidos internacionalmente nos estudos métricos da informação. Em nível de países, a 
realidade mostra que os agradecimentos continuam sendo um fenômeno social anglo-americano. O número elevado de pesquisadores identificados como autores nas publicações sobre agradecimentos pode não representar um alto grau de conexão em rede, mas retratam vínculos colaborativos factuais de natureza intra-institucional.

As descobertas científicas alcançadas até o momento simbolizam 0 esforço desses pesquisadores em um campo temático que não é novo, mas que está em transformação a partir do surgimento de novas oportunidades para obtenção de dados sobre agradecimentos. Sugere-se a ampliação dos estudos métricos da informação sobre agradecimentos na ciência, analisando por meio de indicadores de impacto, as características dos documentos citantes em relação às variáveis: ano de publicação, idioma, tipologia documentária, categoria de assunto, periódico, instituição e país dos autores. Outras bases internacionais como a Scopus podem ser consideradas para coleta de dados, visando uma compreensão mais ampla acerca do fenômeno em questão. Estudos de cocitação podem ser interessantes para analisar a frequência com que pares de documentos são citados conjuntamente em uma publicação, buscando identificar o grau de proximidade temática em nível de autores.

\section{REFERÊNCIAS}

ÁLVAREZ-BORNSTEIN, B.; MORILLO, F.; BORDONS, M. Funding acknowledgments in the Web of Science: completeness and accuracy of collected data. Scientometrics, Amsterdam, v. 112, n. 3, p. 1793-1812, 2017.

BEN-DAVID, J. Introdução. In: Sociologia da Ciência. Rio de Janeiro: Ed. FGV, 1975. p. 1-32.

BOURDIEU, P. Os usos sociais da ciência: por uma sociologia clínica do campo científico. São Paulo: Editora UNESP, 2004.

BOZEMAN, B.; LEE, S. The impact of research collaboration on scientific productivity. In: ANNUAL MEETING OF THE AMERICAN ASSOCIATION FOR THE ADVANCEMENT OF SCIENCE, 2003, Denver. Proceedings [...] Denver: AAAS, 2003. 
CRONIN, B. Let the credits roll: a preliminary examination of the role played by mentors and trusted assessors in disciplinary formation. Journal of

Documentation, v. 47, n. 3, p. 227-239, 1991.

CRONIN, B. Acknowledgement trends in the research literature of information sciences. Journal of Documentation, v. 57, n. 3, p. 329-464, may 2001.

CRONIN, B.; OVERFELT, K. The scholar's courtesy: A survey of acknowledgement behaviour. Journal of Documentation, v. 50, n. 3, p. 165196, 1994.

CRONIN, B.; WEAVER, S. The praxis of acknowledgement: from bibliometrics to influmetrics. Revista Española de Documentación Científica, Madrid, v. 18, n. 2, p. 172-177, 1995.

CRONIN, B.; MCKENZIE, G.; STIFFLER, M. Patterns of acknowledgement. Journal of Documentation, v. 48, n. 2, p. 107-253, jun. 1992.

CRONIN, B.; MCKENZIE, G.; RUBIO, L. The norms of acknowledgement in four humanities and social sciences disciplines. Journal of Documentation, $v$. 49, n. 1, p. 1-102, mar. 1993.

CRONIN, B.; SHAW, D.; LA BARRE, K. A cast of thousands: co-authorship and sub-authorship collaboration in the twentieth century as manifested in the scholarly journal literature of Psychology and Philosophy. Journal of the Association for Information Science and Technology, v. 54, n. 9, p. 855871, jul. 2003.

CRONIN, B.; SHAW, D.; LA BARRE, K. Visible, less visible, and invisible work: patterns of collaboration in 20th century Chemistry. Journal of the American Society for Information Science and Technology, v. 55, n. 2, p. 160-168, 2004.

DÍAZ-FAES, A. A.; BORDONS, M. Making visible the invisible through the analysis of acknowledgements in the humanities. Aslib Journal of Information Management, v. 69, n. 5, p. 576-590, 2017.

GILES, C. L.; COUNCILL, I. G. Who gets acknowledged: measuring scientific contributions through automatic acknowledgment indexing. Proceedings of the National Academy of Sciences of the United States of America, v. 101, n. 51, p. 17599-17604, 2004.

HAYASHI, M. C. P. I. Evidências bibliométricas do reconhecimento científico em resenhas e entrevistas: notas teóricas e modelo de análise. RDBCl:

Revista Digital de Biblioteconomia e Ciência da Informação, Campinas, v. 18, p. 1-31, 2020.

HAYASHI, M. C. P. I. Agradecimentos em artigos científicos: o ponto de vista de pesquisadores. Prisma.com, Porto, n. 37, p. 55-70, 2018. 
HAYASHI, M. C. P. I.; BELLO, S. F. Presença dos agradecimentos em um periódico da área de Saúde. Em Questão, Porto Alegre, v. 20, n. 3, edição especial, p. 166-193, 2014.

HEFFNER, A. Funded research, multiple authorship, and subauthorship collaboration in four disciplines. Scientometrics, Amsterdam, v. 3, n. 1, p. 5-12, 1981.

HIRSCH, J. E. An index to quantify an individual's scientific research output. Proceedings of the National Academy of Sciences of the United States of America, v. 102, n. 46, p. 16569-16572, nov. 2005.

HOCHMAN, G. A ciência entre a comunidade e o mercado: leituras de Kuhn, Bourdieu, Latour e Knorr-Cetina. In: PORTOCARRERO, V. (org.). Filosofia, história e sociologia das ciências: abordagens contemporâneas. Rio de Janeiro: Fiocruz, 1994. p. 199-231.

HYLAND, K. Dissertation acknowledgements: the anatomy of a Cinderella genre. Written Communication, v. 20, n. 3, p. 242-268, 2003.

KATZ, J. S. Geographical proximity and scientific collaboration. Scientometrics, Amsterdam, v. 31, n. 1, p. 31-43, 1994.

KATZ, J. S.; MARTIN, B. R. What is research collaboration? Research Policy, Amsterdam, n. 26, p. 1-18, 1997.

KNORR-CETINA, K. D. Scientific communities or transepistemic arenas of research? A critique of quasi-economic models of science. Social Studies of Science, v. 12, n. 1, p. 101-130, 1982.

KRETSCHMER, H.; ROUSSEAU, R. Author inflation leads to a breakdown of Lotka's law. Journal of the American Society for Information Science and Technology, New York, v. 52, n. 8, p. 610-614, 2001.

KUHN, T. S. A estrutura das revoluções científicas. São Paulo: Perspectiva, 1997.

LATOUR, B.; WOOLGAR, S. A vida de laboratório: a produção dos fatos científicos. Rio de Janeiro: Relume Dumará, 1997.

MCCAIN, K. W. Communication, competition, and secrecy: the production and dissemination of research-related information in Genetics. Science, Technology, \& Human Values, v. 16, n. 4, p. 491-516, 1991.

MERTON, R. K. Sociologia - teoria e estrutura. São Paulo: Mestre Jou, 1968.

MERTON, R. K. O efeito Mateus na ciência II. A vantagem cumulativa e o simbolismo da propriedade intelectual. 1988. In: MARCOVICH, A.; SHINN, T. (org.). Ensaios de Sociologia da Ciência. São Paulo: Editora 34, 2013. p. 199-231. 
PATEL, N. Collaboration in the professional growth of American Sociology.

Social Science Information, v. 12, n. 6, p. 77-92, 1973.

PAUL-HUS, A.; DESROCHERS, N.; PECOSKIE, J.; LARIVIÈRE, V.

Acknowledgment research genealogy for today's quantified academia. In:

ANNUAL CONFERENCE OF THE CANADIAN ASSOCIATION FOR

INFORMATION SCIENCE, 43., 2015, Ottawa. Proceedings [...] Ottawa: CAIS, 2015.

PAUL-HUS, A.; DESROCHERS, N.; COSTAS, R. Characterization, description, and considerations for the use of funding acknowledgement data in Web of Science. Scientometrics, Amsterdam, v. 108, n. 1, p. 167-182, 2016.

SALAGER-MEYER, F.; ARIZA, M. Á. A.; BERBESÍ, M. P. "Backstage solidarity" in Spanish and English written medical research papers: publication context and the acknowledgment paratext. Journal of the American Society for Information Science and Technology, v. 60, n. 2, p. 307-317, 2009.

SALAGER-MEYER, F.; ALCARAZ-ARIZA, M. Á.; LUZARDO BRICEÑO, M.; JABBOUR, G. Scholarly gratitude in five geographical contexts: a diachronic and cross-generic approach of the acknowledgment paratext in medical discourse (1950-2010). Scientometrics, v. 86, n. 3, p. 763-784, 2011.

\title{
SCIENTOMETRIC INDICATORS OF SCIENTIFIC OUTPUT AND COLLABORATION ON THE ACKNOWLEDGMENTS THEMATIC (1980-2019)
}

\begin{abstract}
Objective: It investigates characteristics of worldwide literature about the acknowledgments thematic from the 1980-2019 period, indexed on the Web of Science (WoS) database through scientometric indicators of scientific output and collaboration. Methodology: It characterized as an exploratory and descriptive study which employs quantitative techniques (scientometric) to analyze all the papers covered by the Information Science \& Library Science subject category, using Bibexcel and Pajek softwares. Results: The distribution of the 105 papers about acknowledgments reveals a steady growth tendency during this period. The significant increase since 2009 may have been a result of the work on automatic harvest and processing of acknowledgment data started by the WoS in 2008. Foreign journals written in English lead the productivity and impact ranking, emphasizing the internationalization and quality of the scientific output. The indicators point to two generations of researchers on the acknowledgments thematic, the first standing out in analysis of usage, frequency and prevalence of acknowledgment support type; and the second in funding information analysis. Although the co-authorship identified in the papers might not represent a high level of network connections, they portray factual collaborative links of an intra-institutional nature. Conclusions: It concluded that the findings achieved so far symbolize the effort of these researchers in a thematic field that is not new, but that is changing due to the arise of new opportunities to obtain acknowledgments data.
\end{abstract}


Descriptors: Acknowledgments. Scientific output. Scientific collaboration. Indicators scientometrics. Scientometric.

\title{
INDICADORES CIENCIOMÉTRICOS DE PRODUCCIÓN Y COLABORACIÓN CIENTÍFICA SOBRE EL TEMA AGRADECIMIENTOS (1980-2019)
}

\begin{abstract}
RESUMEN
Objetivo: Se investigan las características de la literatura mundial sobre el tema de los agradecimientos del período 1980-2019 indexada en la base de datos Web of Science (WoS) a través de indicadores cienciométricos de producción y colaboración científica. Metodología: Se caracteriza como un estudio exploratorio y descriptivo que utilizó técnicas cuantitativas (cienciometría) para analizar todos los artículos cubiertos por la categoría de asunto Information Science \& Library Science, con la ayuda de los softwares Bibexcel y Pajek. Resultados: La distribución de los 105 artículos sobre agradecimientos revela una tendencia de crecimiento constante durante el período. El aumento significativo desde 2009 puede haber sido consecuencia del trabajo de captura y procesamiento automático de datos sobre agradecimientos iniciado por la WoS en 2008. Las revistas extranjeras en idioma inglés lideran el ranking de productividad e impacto, destacando la internacionalización y la calidad de la producción científica. Los indicadores apuntan para dos generaciones de investigadores en el tema de los agradecimientos, destacándose la primera en los análisis de uso, frecuencia y prevalencia del tipo de apoyo agradecido y la segunda en el análisis de información sobre financiamiento. Aunque las coautorías identificadas en los artículos puedan no representar un alto grado de conexión en red, retratan vínculos colaborativos factuales de naturaleza intrainstitucional. Conclusiones: Se concluye que los descubrimientos logrados hasta ahora simbolizan el esfuerzo de estos investigadores en un campo temático que no es nuevo, pero que está en transformación debido a la aparición de nuevas oportunidades para obtener datos sobre agradecimientos.
\end{abstract}

Descriptores: Agradecimientos. Productividad científica. Colaboración científica. Indicadores cienciométricos. Cienciometría.

Recebido em: 03.04.2021

Aceito em: 13.12.2021 